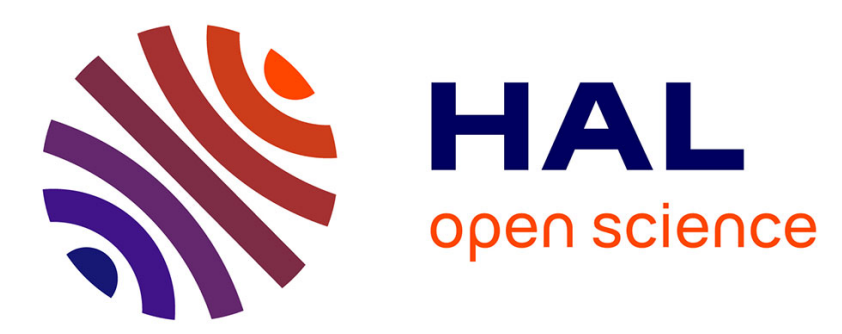

\title{
The excitation of CNCN in the interstellar medium: hyperfine resolved rate coefficients and non-LTE modelling
}

D. Ndaw, Cheikh T Bop, G. Dieye, N. A. Boye Faye, François Lique

\section{- To cite this version:}

D. Ndaw, Cheikh T Bop, G. Dieye, N. A. Boye Faye, François Lique. The excitation of CNCN in the interstellar medium: hyperfine resolved rate coefficients and non-LTE modelling. Monthly Notices of the Royal Astronomical Society, 2021, 503 (4), pp.5976-5983. 10.1093/mnras/stab775 . hal-03267826

\author{
HAL Id: hal-03267826 \\ https://hal.science/hal-03267826
}

Submitted on 15 Oct 2021

HAL is a multi-disciplinary open access archive for the deposit and dissemination of scientific research documents, whether they are published or not. The documents may come from teaching and research institutions in France or abroad, or from public or private research centers.
L'archive ouverte pluridisciplinaire HAL, est destinée au dépôt et à la diffusion de documents scientifiques de niveau recherche, publiés ou non, émanant des établissements d'enseignement et de recherche français ou étrangers, des laboratoires publics ou privés. 


\title{
The excitation of $\mathrm{CNCN}$ in the interstellar medium: Hyperfine resolved rate coefficients and non-LTE modeling
}

\author{
D. Ndaw ${ }^{1}$, C. T. Bop ${ }^{1,2 \star}$, G. Dieye ${ }^{3}$, N. A. Boye Faye ${ }^{1}$ and F. Lique ${ }^{2}$ \\ ${ }^{1}$ Laboratoire Atomes Lasers, Département de Physique, Faculté des Sciences et Techniques, Université Cheikh Anta Diop, Dakar, 5005, Senegal \\ ${ }^{2}$ Univ Rennes, CNRS, IPR (Institut de Physique de Rennes) - UMR 6251, F-35000 Rennes, France \\ ${ }^{3}$ Laboratoire pour l'utilisation des rayons X, Département de Physique, Faculté des Sciences et Techniques, Université Cheikh Anta Diop, Dakar, 5005, \\ Senegal
}

Accepted XXX. Received YYY; in original form ZZZ

\begin{abstract}
The recent detections of $\mathrm{CNCN}$ and $\mathrm{HNCCN}^{+}$are seen as further evidence of the large abundance of NCCN in the interstellar medium. The accurate determination of the abundance of these chemically related compounds from the observational spectra requires the prior calculation of collisional rate coefficients. In this work, we aimed at computing hyperfine resolved rate coefficients for the $\mathrm{CNCN}-\mathrm{He}$ collisional system. First, we determined a new potential energy surface for the $\mathrm{CNCN}-\mathrm{He}$ van der Waals complex from which we computed rotationally resolved excitation cross sections for energies up to $800 \mathrm{~cm}^{-1}$ using the quantum mechanical close-coupling approach. Then, hyperfine resolved transitions between the 30 lowlying pure rotational levels of $\mathrm{CNCN}$ were computed for temperatures ranging from $5 \mathrm{~K}$ to $150 \mathrm{~K}$ using an improved infinite order sudden approach. The analysis of the scattering results showed a propensity rule in favor of $\Delta j=\Delta F_{1}=\Delta F$ for the hyperfine transitions and a slight dominance of the odd $\Delta j$ transitions. Using these data, we carried out non-LTE radiative transfer calculations to simulate the excitation of $\mathrm{CNCN}$ in molecular clouds and to constrain the physical conditions of cold dark clouds. Preliminary results showed that the abundance of $\mathrm{CNCN}$ derived from observational spectra has to be revisited using these new collisional data.
\end{abstract}

Key words: ISM: molecules - molecular data - molecular processes - ISM : abundances

\section{INTRODUCTION}

Cyanogen $(\mathrm{NCCN})$ is the simplest and smallest molecule among the dicyanopolyynes $\left(\mathrm{N} \equiv \mathrm{C}-(\mathrm{C} \equiv \mathrm{C})_{n}-\mathrm{C} \equiv \mathrm{N}, n=0,1,2 \ldots\right)$ family. Despite the high spectral and spatial resolution of newer telescopes, NCCN cannot be directly observed through its rotational spectrum as this molecule has no permanent electric dipole moment. Therefore various strategies have been suggested to investigate the NCCN abundance in astrophysical environments.

First, NCCP, a chemical analogue of cyanogen which has a permanent electric dipole moment of 6.347 D (Puzzarini \& Cazzoli 2009), was tentatively identified towards the carbon-star envelope IRC+10216 with the IRAM 30 m telescope (Agúndez et al. 2014). This detection, once definitively confirmed, may be a proof of the presence of NCCN towards the IRC+10216 circumstellar envelope since the observations of phosphorous containing molecules in this source were all preceded by the identification of the corresponding N-bearers.

Then, the detection of protonated cyanogen, $\mathrm{HNCCN}^{+}$, towards TMC-1 and L483 (Agúndez et al. 2015) can also be seen as a proof regarding the NCCN presence in the interstellar medium

^ cheikhtidiane.bop@ucad.edu.sn
(ISM) (Petrie et al. 2003). Indeed, $\mathrm{HNCCN}^{+}$is likely produced by proton transfer from $\mathrm{HCO}^{+}$and $\mathrm{H}_{3}^{+}$to $\mathrm{NCCN}$. Chemical models predict a $\left[\mathrm{HNCCN}^{+}\right] /[\mathrm{NCCN}]$ abundance ratio of $\sim 10^{-4}$ in cold dark clouds (Agúndez et al. 2015). Therefore, deriving the $\mathrm{HNCCN}^{+}$ column density from its observations (Agúndez et al. 2015; Vastel et al. 2019) could lead to better insights on the cyanogen abundance in the ISM.

Finally, the NCCN abundance can also be probed using one of its metastable isomer, the isocyanogen (CNCN). This latter was detected in space for the first time towards the L483 class 0 protostar host and tentatively towards the TMC-1 molecular clouds (Agúndez et al. 2018b). More recently, Vastel et al. (2019) reported detection of the $j=8 \rightarrow 7$ and $10 \rightarrow 9$ isocyanogen emission lines from the L1544 prototypical prestellar core. Due to the lack of chemical model relating the abundances of $\mathrm{CNCN}$ and NCCN, Agúndez et al. (2018b) supposed a $[\mathrm{CNCN}] /[\mathrm{NCCN}]$ abundance ratio similar to $[\mathrm{HCCNC}] /[\mathrm{HCCCN}]$, these two pairs of isomer sharing some structural characteristics, namely the $-\mathrm{CNC}$ and $-\mathrm{CCN}-$ groups (Petrie et al. 2003). Since these four molecules are detected in the source, presumably under the same physical conditions, the $[\mathrm{HCCNC}] /[\mathrm{HCCCN}]$ abundance ratio together with the population of CNCN may help in estimating the density of NCCN in space.

The use of the protonated cyanogen column desnity (Agún- 
dez et al. 2015) would lead to a NCCN column density of $(8.6 \pm 4.4) \times 10^{14} \mathrm{~cm}^{-2}$ in TMC-1 and $(3.9 \pm 1.8) \times 10^{14} \mathrm{~cm}^{-2}$ in L483. On the other hand, the use of the CNCN column density (Agúndez et al. 2018b) along with the $[\mathrm{HCCNC}] /[\mathrm{HCCCN}]$ ratio (Agúndez et al. 2019; Cernicharo et al. 2020) would yield to a NCCN column density of $(6.9 \pm 0.7) \times 10^{13} \mathrm{~cm}^{-2}$ and $\sim 1.2 \times 10^{14}$ $\mathrm{cm}^{-2}$ in TMC-1 and L483, respectively. The abundance derived using $\mathrm{HNCCN}^{+}$is greater than that obtained from $\mathrm{CNCN}$ observations by factors of $\sim 12$ and $\sim 3$ in TMC-1 and L483, respectively. Therefore, better insights into the $\mathrm{HNCCN}^{+}$and $\mathrm{CNCN}$ abundances in cold dark clouds are necessary to depict more robust conclusions since the two way to estimate the NCCN abundance lead to quite different values for the same medium.

Molecular abundances must be derived using radiative transfer models. These latter require some basic inputs such as Einstein coefficients which should be associated to collision rate coefficients when local thermodynamic equilibrium (LTE) is not reach. In fact, LTE conditions are rarely fulfilled in the ISM which implies the need of prior computation of collision data (Roueff \& Lique 2013). Bop et al. (2018) reported excitation rate coefficients of $\mathrm{HNCCN}^{+}$ induced by collision with $\mathrm{He}$ for temperatures up to $100 \mathrm{~K}$.

In parallel, Ben Abdallah et al. (2018) computed rotational excitation rate coefficients of $\mathrm{CNCN}$ due to collision with He considering only the 16 low-lying rotational levels. Modeling the $j=8 \rightarrow 7$, $9 \rightarrow 8$ and $10 \rightarrow 9$ CNCN lines detected towards L483, L1544 and TMC-1 (Agúndez et al. 2018b; Vastel et al. 2019) using radiative transfer models probably requires to determine the population of higher rotational levels than those considered by Ben Abdallah et al. (2018). In addition, due to the nuclear spin of the nitrogen atoms, the $\mathrm{CNCN}$ rotational states are split into hyperfine levels that were not considered in the work of Ben Abdallah et al. (2018). Hence, in this paper, we revisited the excitation of $\mathrm{CNCN}$ induced by collision with helium and extend the work of Ben Abdallah et al. (2018) to (i) a higher rotational basis ( $j$ up to 29 instead of 15) and (ii) by resolving the hyperfine structure due to the nuclear spin of the nitrogen atoms in the scattering calculations.

The use of $\mathrm{He}$ as projectile comes from the fact that $\mathrm{He}$ is expected to be a reasonable template for para- $\mathrm{H}_{2}\left(j_{2}=0\right)\left(j_{2}\right.$ being the $\mathrm{H}_{2}$ rotational quantum number). Indeed, $\mathrm{H}_{2}\left(j_{2}=0\right)$ is the most abundant species in the ISM (especially in cold dark clouds where $\mathrm{CNCN}$ was detected) and then the dominant collision partner. As $\mathrm{H}_{2}\left(j_{2}=0\right)$, He is a closed shell compound with two-valence electrons and a spherical structure. The He-rate coefficients multiplied by a scaling factor such as the square root of the reduced mass ratio can be used to roughly derive $\mathrm{H}_{2}\left(j_{2}=0\right)$-rate coefficients (Lique et al. 2008). It is worth mentioning that this approximation has limits especially in the case of charged molecules and hydrides. However, the pioneer work of Wernli et al. (2007) on $\mathrm{HC}_{3} \mathrm{~N}-\mathrm{He}$ and $\mathrm{HC}_{3} \mathrm{~N}-\mathrm{H}_{2}$ which showed an average ratio between the $\mathrm{H}_{2}\left(j_{2}\right.$ $=0$ )- and He-rate coefficients of $\sim 1.4$ lets us expect He to be a reasonable template for $\mathrm{H}_{2}$ in the case of the $\mathrm{CNCN}$ molecule.

This paper is organized as follows: Section 2 presents the computational details. The results are presented and discussed in Section 3. Radiative transfer calculations and astrophysical implications are shown in Section 4. In Section 5, are given concluding remarks.

\section{COMPUTATIONAL DETAILS}

\subsection{Potential energy surface and analytical fit}

In the following, we consider the $\mathrm{CNCN}$ molecule as a rod-like shape rigid-rotor. Two degrees of freedom are then needed to describe the

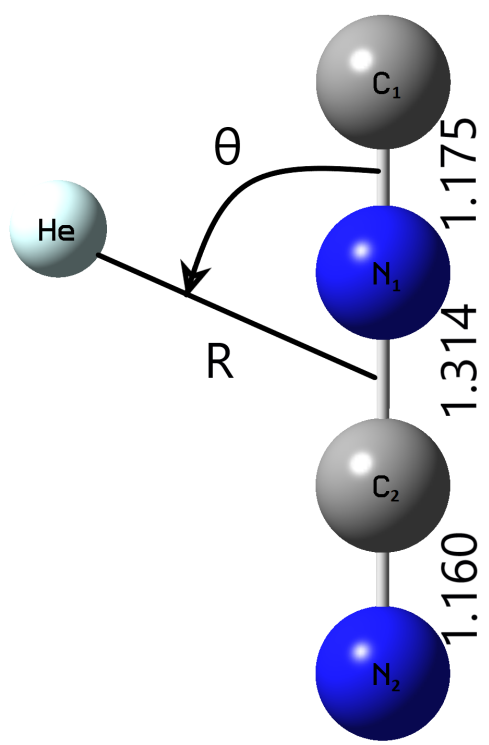

Figure 1. Body-fixed Jacobi coordinate system for the CNCN-He interaction. The parameters are given in $\AA$.

potential energy surface (PES) in the Jacobi coordinate system (see Fig. 1), namely the distance $(R)$ separating $\mathrm{He}$ and the center of mass of CNCN and the scattering angle $(\theta)$ between the CNCN back-bone axis and the colliding axis. The structure of the molecule is described using the experimental ground vibrational geometry (Stroh \& Winnewisser 1989). Typically, $\mathrm{r}_{\mathrm{C}_{1} \mathrm{~N}_{1}}, \mathrm{r}_{\mathrm{N}_{1} \mathrm{C}_{2}}$ and $\mathrm{r}_{\mathrm{C}_{2} \mathrm{~N}_{2}}$ were set to $1.175 \AA, 1.314 \AA$ and $1.160 \AA$, respectively. Therefore, the interacting system is linear when $\theta=0^{\circ}\left(\mathrm{He} \cdots \mathrm{C}_{1}-\mathrm{N}_{1}-\mathrm{C}_{2}-\mathrm{N}_{2}\right)$ and $\theta=180^{\circ}\left(\mathrm{C}_{1}-\mathrm{N}_{1}-\mathrm{C}_{2}-\mathrm{N}_{2} \cdots \mathrm{He}\right)$.

The potential was constructed using $\mathrm{C}_{s}$ point group with the radial coordinate $R$ varying from $\sim 2$ to $50 \AA$. The step was set to $\sim 0.1 \AA$ for $2 \leq R \leq 6 \AA, 0.25 \AA$ for $6.25 \leq R \leq 8 \AA, 0.5 \AA$ for $8.5 \leq R \leq 10 \AA, 1 \AA \AA$ for $11 \leq R \leq 15 \AA$. In addition, the potential was calculated at $R=50 \AA$ for size consistency reason (see the paragraph below). Concerning the scattering angle, it was uniformly spanned from 0 to $180^{\circ}$ with a step of $10^{\circ}$. Therefore, 931 ab initio points were used to generate the PES.

All calculations ${ }^{1}$ were performed using the explicitly correlated coupled cluster approach with single, double, and non-iterative triple excitation (CCSD(T)-F12) approach developed by Knizia et al. (2009) supplemented by the augmented-correlation consistent polarized Valence Triple Zeta Gaussian basis set (aug-cc-pVTZ) of Dunning Jr (1989) as implemented in the MOLPRO molecular package version 2010 (Werner et al. 2010). The use of the CCSD(T)-F12 method together with an aug-cc-pVTZ basis set is expected to lead to highly accurate PES. Indeed, the accuracy of such approach was assessed in the literature with respect to standard coupled cluster in conjunction with aug-cc-pVnZ (n being triple, quadruple and quintuple) and complete basis set extrapolation (Knizia et al. 2009; Lique et al. 2010; Bop et al. 2017a,b).

Due to the size consistency problem of the $\operatorname{CCSD}(\mathrm{T})-\mathrm{F} 12$ method, we deduced the value of the potential obtained at $50 \AA$ to all $a b$ initio points, for each angle. Thus, the potential is constrained to tend asymptotically to zero at $50 \AA$. Errors generated

1 Note that a PES of similar accuracy has been published by Ben Abdallah et al. (2018) after the start of this research project. 
by the basis set superposition were corrected following the Boys \& Bernardi (1970) counterpoise procedure (see eq. 1):

$$
V(\mathrm{R}, \theta)=E_{\mathrm{CNCN}-\mathrm{He}}(\mathrm{R}, \theta)-E_{\mathrm{CNCN}}(\mathrm{R}, \theta)-E_{\mathrm{He}}(\mathrm{R}, \theta)
$$

As required by the MOLSCAT computer code which was used for scattering calculations, we expand the potential over the Legendre polynomial functions (see eq. 2).

$V(R, \theta)=\sum_{\lambda=0}^{\lambda_{\max }} V_{\lambda}(R) P_{\lambda}(\cos \theta)$

$V_{\lambda}$ and $P_{\lambda}$ represent the radial coefficients and the angular functions, respectively.

Because of the large size of the $\mathrm{CNCN}$ molecule, the expansion over Legendre polynomial functions is difficult to perform at short $R$-distances. To overcome such a difficulty, a large number of radial terms (35 $\lambda$-values) were needed. As only 19 angles were used to generate the $a b$ initio potential, we first interpolated the data with the cubic spline routine. The relative error difference between the analytical PES and the ab initio data is less than $0.8 \%$ for $\mathrm{R}$ $\geq 3.5 \AA$ and $1.3 \%$ for the whole 931 grid points. The long-range $(R \geq 15 \AA)$ interaction potential was obtained by extrapolating the radial coefficients using the POTENL routine implemented in the MOLSCAT code.

$$
V_{\lambda}(R \geq 15 \AA)=\sum_{n} \frac{C_{n}}{R^{n}}
$$

The coefficients of the inverse exponent expansion, $C_{n}$, are determined using the last radial terms (i.e. $R$ approaching $15 \AA$ ).

\subsection{Cross-sections and rate coefficients}

The main goal of this paper is to obtain hyperfine structure resolved $\mathrm{CNCN}-\mathrm{He}$ rate coefficients. However, considering the 30 low-lying rotational levels along with the nuclear spin of the two nitrogen atoms of CNCN leads to 262 hyperfine levels. Performing quantum scattering calculations with such a large number of energy levels would be prohibitive both in terms of CPU time and memory. Hence, we decided to compute, first, rotationally resolved rate coefficients using the close-coupling method (Arthurs \& Dalgarno 1960). Hyperfine structure resolved rate coefficients were then derived from the rotational ones. Therefore, the collisional process we are interested in can be summarized as follows:

$$
\begin{aligned}
\mathrm{CNCN}(j)+\mathrm{He} & \rightarrow \mathrm{CNCN}\left(j^{\prime}\right)+\mathrm{He} \\
\mathrm{CNCN}\left(j, F_{1}, F\right)+\mathrm{He} & \rightarrow \mathrm{CNCN}\left(j^{\prime}, F_{1}^{\prime}, F^{\prime}\right)+\mathrm{He},
\end{aligned}
$$

where $F_{1}$ and $F$ are defined as $|j-I| \leq F_{1} \leq(I+j)$ and $\left|F_{1}-I\right| \leq$ $F \leq\left(F_{1}+I\right)$, respectively with $I=1$ being the nuclear spin of nitrogen.

The state-to-state rotational cross-sections were calculated using the MOLSCAT code (Hutson \& Green 1994). The coupled equations were solved with the modified log derivative-airy integrator of Alexander \& Manolopoulos (1987). The size of the rotational basis, was varied from 15 to 37 leading to include all open channels and at least 7 closed channels. The STEPS parameter was progressively decreased from 30 to 10 to ensure the integration step to be small enough for good convergence. The radial wave function was integrated from 1.5 to at least $25 \AA$. Using these parameters in the cross-section calculations, we spanned the energy range from 0.4 to $800 \mathrm{~cm}^{-1}$ with a step size chosen as follows: $0.2 \mathrm{~cm}^{-1}$ for energies below $250 \mathrm{~cm}^{-1}, 1 \mathrm{~cm}^{-1}$ from 251 to $300 \mathrm{~cm}^{-1}, 5 \mathrm{~cm}^{-1}$ from 305 to $400 \mathrm{~cm}^{-1}, 10 \mathrm{~cm}^{-1}$ from 410 to $500 \mathrm{~cm}^{-1}, 20 \mathrm{~cm}^{-1}$ from 520 $\mathrm{cm}^{-1}$ to $600 \mathrm{~cm}^{-1}$ and to $50 \mathrm{~cm}^{-1}$ from $650 \mathrm{~cm}^{-1}$ to $800 \mathrm{~cm}^{-1}$. The spectroscopic constants used in the rotational basis construction $B_{e}$ and $D_{e}$ are $0.1726 \mathrm{~cm}^{-1}$ and $0.223 \times 10^{-7} \mathrm{~cm}^{-1}$ (Botschwina 1994), respectively and the reduced mass $\mu$ of the colliding system is 3.7167 a.m.u.

Using the cross-sections calculated for total energies up to 800 $\mathrm{cm}^{-1}$, we are able to derive close-coupling rate coefficients $\left(k_{j \rightarrow j^{\prime}}^{C C}\right)$ for transitions between the first 30 low-lying pure rotational levels of $\mathrm{CNCN}$ and for temperatures up to $150 \mathrm{~K}$. In practice, we retrieved these data by thermally averaging the inelastic cross-sections $\sigma\left(E_{k}\right)$ over the Maxwell-Boltzmann velocity distribution,

$k_{j \rightarrow j^{\prime}}^{C C}(T)=\left(\frac{8}{\pi \mu \beta}\right)^{1 / 2} \beta^{2} \int_{0}^{\infty} E_{k} \sigma\left(E_{k}\right) e^{-\beta E_{k}} d E_{k}$

where $\beta=1 / k_{B} T$ with $k_{B}$ being the Boltzmann constant.

The CNCN hyperfine structure is resolved by first determining the infinite order sudden (IOS) rate coefficients for the pure rotational $\left(k_{j \rightarrow j^{\prime}}^{I O S}\right)$ and hyperfine $\left(k_{j, F_{1}, F \rightarrow j^{\prime}, F_{1}^{\prime}, F^{\prime}}^{I O S}\right)$ transitions. These latter are related to the fundamental IOS rates $\left(k_{0 \rightarrow L}\right)$ as follows (Goldflam et al. 1977) :

$$
k_{j \rightarrow j^{\prime}}^{I O S}(T)=\left(2 j^{\prime}+1\right) \sum_{L}\left(\begin{array}{lll}
j^{\prime} & j & L \\
0 & 0 & 0
\end{array}\right)^{2} k_{0 \rightarrow L}(T)
$$

$$
\begin{aligned}
& k_{j, F_{1}, F \rightarrow j^{\prime}, F_{1}^{\prime}, F^{\prime}}^{I O S}=(2 j+1)\left(2 j^{\prime}+1\right)\left(2 F_{1}+1\right)\left(2 F_{1}^{\prime}+1\right)\left(2 F^{\prime}+1\right) \times \\
& \sum_{L}\left(\begin{array}{lll}
j^{\prime} & j & L \\
0 & 0 & 0
\end{array}\right)^{2}\left\{\begin{array}{ccc}
j & j^{\prime} & L \\
F_{1}^{\prime} & F_{1} & I
\end{array}\right\}^{2}\left\{\begin{array}{lll}
F_{1} & F_{1}^{\prime} & L \\
F^{\prime} & F & I
\end{array}\right\}^{2} k_{0 \rightarrow L}(T)
\end{aligned}
$$

In practice, we used the fundamental CC rates instead of the fundamental IOS collision rates as suggested by Neufeld \& Green (1994). Only the data for which $L \leq 29$ are used due to the size of the rotational basis considered in this work. Then, the rate coefficients

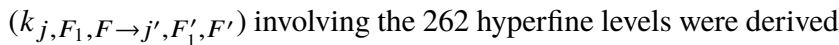
from the spin-free close-coupling scattering data $k_{j \rightarrow j^{\prime}}^{C C}$ (eq. 6), the pure rotational IOS rates (eq. 7) and the hyperfine resolved IOS rates (eq. 8).

$$
k_{j, F_{1}, F \rightarrow j^{\prime}, F_{1}^{\prime}, F^{\prime}}(T)=\frac{k_{j, F_{1}, F \rightarrow j^{\prime}, F_{1}^{\prime}, F^{\prime}}^{I O S}(T)}{k_{j \rightarrow j^{\prime}}^{I O S}(T)} \times k_{j \rightarrow j^{\prime}}^{C C}(T)
$$

\section{RESULTS}

We display in Fig 2 the contour plots of the CNCN-He PES. This highly anisotropic PES presents a relatively shallow global minimum of $55.23 \mathrm{~cm}^{-1}$ located in the T-shape geometry $\left(\theta=90^{\circ}\right)$ at $R=3.13 \AA$. This finding is in good agreement with the results of Ben Abdallah et al. (2018) who reported a well depth of $56.42 \mathrm{~cm}^{-1}$ at a geometry $(R, \theta)$ of $\left(3.15 \AA, 90^{\circ}\right)$. The difference $(\sim 2 \%)$ between the two global minima likely results from the different MOLPRO versions and/or convergence criteria used in the PES calculations as well as the fitting procedures.

Fig. 3 shows rotational de-excitation cross-sections of $\mathrm{CNCN}$, induced by collision with $\mathrm{He}$, as a function of the kinetic energy. Both Feshbach and shape resonances can be seen below $20 \mathrm{~cm}^{-1}$. The Feshbach resonances can be associated to the formation of quasi-bond states when the projectile is temporary trapped in the 


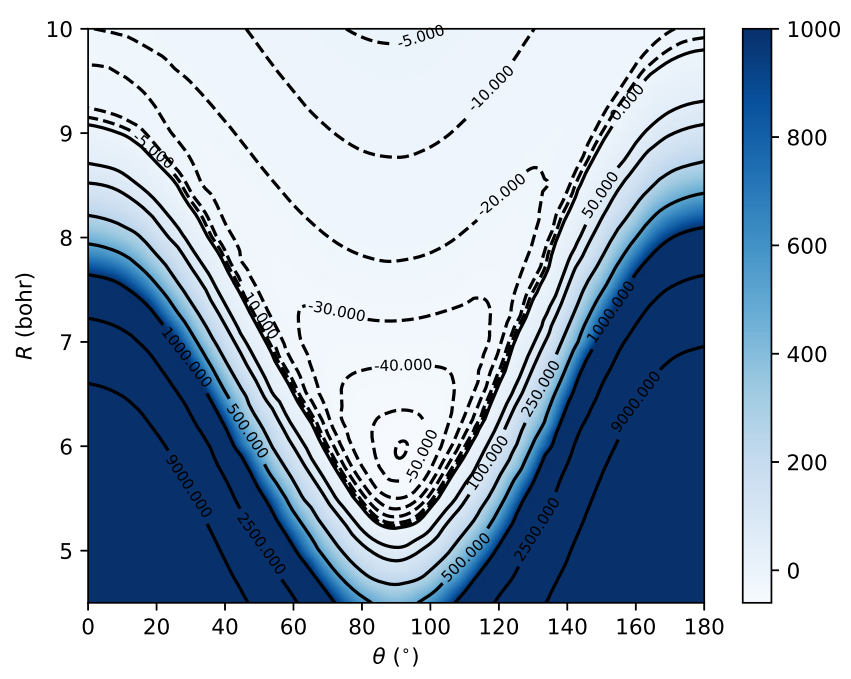

Figure 2. Contour plots of the CNCN-He potential energy surface (in unit of $\mathrm{cm}^{-1}$ ) as a function of $R$ and $\theta$. The zero energy corresponds to the dissociation limit of the monomers. The data in the color map scale are in $\mathrm{cm}^{-1}$.

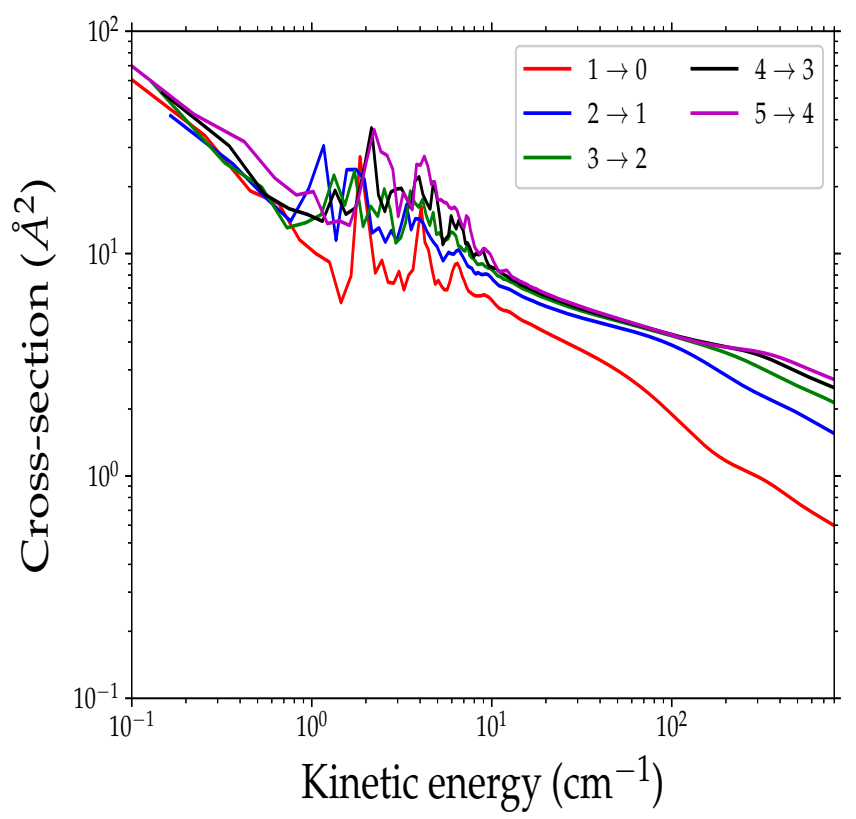

Figure 3. State-to-state inelastic cross-sections of $\mathrm{CNCN}$ induced by collision with $\mathrm{He}$ as a function of the kinetic energy.

potential well while the shape resonances are caused by tunneling effects through the centrifugal energy barrier. These resonances persist for all $\Delta j=1$ transitions (even when involving higher rotational levels) which confirms the importance of the dense energy grid that we used in the calculation (see section 2.2).

In Fig. 4, we plot rate coefficients for the pure rotational de-excitations along with some hyperfine transitions labeled as $j_{F_{1}, F} \rightarrow j_{F_{1}^{\prime}, F^{\prime}}^{\prime}$. First of all, the rate coefficients agree very well with the data of Ben Abdallah et al. (2018), because of the similarity of the PESs and scattering approaches used.

In each panel of Fig. 4, the dominant hyperfine transition is very close to the pure rotational one. This similarity is even better for transitions involving high energy levels. In all panels, the agree-
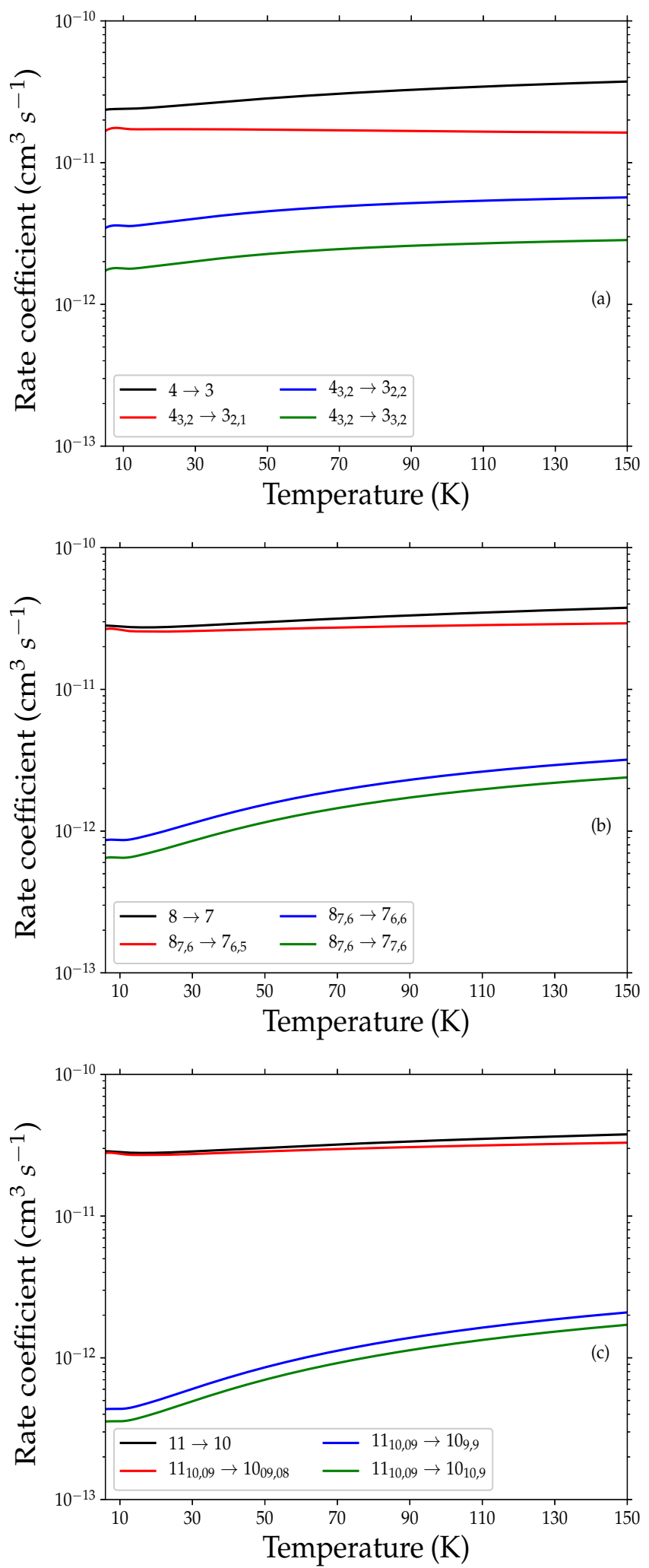

Figure 4. Downward rate coefficients of $\mathrm{CNCN}$ induced by collision with $\mathrm{He}$ as a function of the kinetic temperature. In each panel, the pure rotational and hyperfine transitions are labelled as $j \rightarrow j^{\prime}$ and $j_{F_{1}, F} \rightarrow j_{F_{1}^{\prime}, F^{\prime}}^{\prime}$, respectively 
ment between the dominant hyperfine and pure rotational transitions gradually vanishes with the temperature increase. On the other hand, the weakest hyperfine transitions present large magnitude differences, mainly at low temperature, with respect to the pure rotational ones. Despite the amplitude differences, the rate coefficients behave all similarly. They present a small peak at low temperature and smoothly increase with the temperature increase.

The dependence of the rate coefficients on the final energy levels (e.g. propensity rule) is shown in Fig. 5 for selected temperatures. For the pure rotational de-excitations (panel (a)), no clear propensity rule is found but the dominance is slightly in favor of odd $\Delta j$ transitions at high temperature. The less clear pattern can be attributed to the quasi-symmetric shape of the potential. Concerning the hyperfine transitions shown in panels (b) and (c), rate coefficients for which $\Delta j=\Delta F_{1}$ and $\Delta j=\Delta F_{1}=\Delta F$ absolutely outweigh for all temperatures, respectively. Such behaviours were seen and discussed in previous works (Ben Abdallah et al. 2012; Bop 2019; Balança et al. 2020). At low temperature, the favored hyperfine transitions are at least one order of magnitude greater than the less pronounced ones. The difference gradually decreases with the temperature increase but remains quite important up to at least $100 \mathrm{~K}$

\section{ASTROPHYSICAL IMPLICATIONS}

In interstellar clouds, the emission line intensities are proportional to the population of the upper molecular energy levels. The evolution of these populations depends on radiative and collisional processes which are governed by the Einstein and rate coefficients, respectively. Having these two sets of data allows to perform radiative transfer calculations for the CNCN molecule using a non-LTE approach.

\subsection{The excitation of $\mathrm{CNCN}$}

We performed radiative transfer calculations using the escape probability formalism implemented in the RADEX computer code (Van der Tak et al. 2007). As input, we used the hyperfine rate coefficients computed in this work along with the $\mathrm{CNCN}$ line frequencies $(v)$, energy levels as well as Einstein coefficients, $A\left(j, F 1, F \rightarrow j^{\prime}, F_{1}^{\prime}, F^{\prime}\right)$, downloaded from the Cologne Database for Molecular Spectroscopy (CDMS) portal (Endres et al. 2016). We also treated the $\mathrm{CNCN}$ excitation without considering its hyperfine structure. We derived the Einstein coefficients for the CNCN pure rotational transitions, $A\left(j \rightarrow j^{\prime}\right)$, using eq. 10 as, to the best of our knowledge, these data are missing in the literature.

$$
A\left(j \rightarrow j^{\prime}\right)=\sum_{F^{\prime}, F_{1}^{\prime}} A\left(j, F_{1}, F \rightarrow j^{\prime}, F_{1}^{\prime}, F^{\prime}\right)
$$

In practice, we scaled the $\mathrm{CNCN}-\mathrm{He}$ rate coefficients by a factor of 1.38 to roughly approximate the rate coefficients that para $-\mathrm{H}_{2}$ would induce (Lique et al. 2008).

We determined the brightness $\left(T_{\mathrm{B}}\right)$ and excitation $\left(T_{\mathrm{ex}}\right)$ temperatures for the $j \rightarrow j-1(j=1-11)$ pure rotational emission lines and the associated hyperfine transitions involved in the observed CNCN emission lines (Agúndez et al. 2018b; Vastel et al. 2019).

The background radiation field was set to $2.73 \mathrm{~K}$ which corresponds to the cosmic microwave background temperature $\left(T_{\mathrm{CMB}}\right)$. The line width was fixed at $0.5 \mathrm{~km} \mathrm{~s}^{-1}$, i.e. a value close enough to the average of the full-width at the half-maximum of the observed CNCN spectra (Agúndez et al. 2018b; Vastel et al. 2019) and typical of cold core conditions. To fully cover the gas kinetic temperature of cold dark clouds such as TMC-1, L1544 and L483, the media where $\mathrm{CNCN}$ was detected, we varied the temperature from $5 \mathrm{~K}$ to $25 \mathrm{~K}$. The $\mathrm{H}_{2}$ volume density $(n)$ was gradually increased from $10^{2}$ $\mathrm{cm}^{-3}$ to $10^{8} \mathrm{~cm}^{-3}$ to check the behaviour of the $\mathrm{CNCN}$ excitation under and out of LTE conditions.

We display in Fig. 6 the variation of the $\mathrm{CNCN}$ excitation temperature for the $j=4 \rightarrow 3,8 \rightarrow 7$ and $11 \rightarrow 10$ pure rotational emission lines as well as some corresponding hyperfine transitions as a function of the $\mathrm{H}_{2}$ volume density for selected temperatures. These plots are obtained using a fixed $\mathrm{CNCN}$ column density of $10^{12} \mathrm{~cm}^{-2}$ which corresponds to the typical $\mathrm{CNCN}$ abundance in cold molecular clouds (Agúndez et al. 2018b; Vastel et al. 2019). However, a variation up to an order of magnitude of this quantity does not affect the magnitude of the excitation temperatures because of the optically thin regime. Globally, the excitation temperature increases monotonically with the increase of both the temperature and the $\mathrm{H}_{2}$ density predicting no suprathermal excitation and nor maser effects. Concretely, $T_{\mathrm{ex}}$ is very close to $2.73 \mathrm{~K}$ (i.e. $T_{\mathrm{CMB}}$ ) at low $\mathrm{H}_{2}$ density (a dilute medium dominated by the background radiation field), gradually increases with the increase of the $\mathrm{H}_{2}$ volume density (mostly in the $10^{3}-10^{5} \mathrm{~cm}^{-3}$ density regime) and then tends asymptotically to the kinetic temperature value for $n \geq$ $10^{6} \mathrm{~cm}^{-3}$ where LTE conditions are expected. For the $j=4 \rightarrow 3$ and $j=11 \rightarrow 10$ lines, LTE conditions are fulfilled for $n \geq$ $10^{5} \mathrm{~cm}^{-3}$ and $n \geq 10^{6} \mathrm{~cm}^{-3}$, respectively. As the gas density in cold dark clouds where CNCN was observed is a few $10^{4} \mathrm{~cm}^{-3}$, considering LTE to model the $\mathrm{CNCN}$ abundance is very risky since the density of the medium does not necessarily exceed the critical density.

Concerning the hyperfine structure, the bottom panels show perfect overlaps of the excitation temperatures for transitions involving the same $j$ and $j^{\prime}$, demonstrating that the excitation of CNCN does not present any hyperfine anomalies.

Fig. 7 shows the variation of the brightness temperature for the CNCN $j=4 \rightarrow 3,8 \rightarrow 7$ and $11 \rightarrow 10$ rotational lines as well as the $j_{F_{1}, F}=4_{3,2} \rightarrow 3_{2,1}, 8_{7,6} \rightarrow 7_{6,5}$ and $11_{10,9} \rightarrow 10_{9,8}$ hyperfine lines with the $\mathrm{H}_{2}$ volume density for given temperatures. The brightness temperatures for the hyperfine lines corresponding to $\Delta j \neq \Delta F_{1}$ and/or $\Delta F$ transitions are very weak $\left(T_{\mathrm{B}}<0.1 \mathrm{mK}\right)$ and are therefore not represented in this graph. In all panels, the physical quantity globally increases with the increase of the density and the temperature with an asymptotic behaviour at $n>10^{6} \mathrm{~cm}^{-3}$. A small peak is observed in panels (b) and (b') for $T \geq 17 \mathrm{~K}$ and $2 \times 10^{4} \mathrm{~cm}^{-3} \leq n \leq 6 \times 10^{5} \mathrm{~cm}^{-3}$. In panels (a) and (a'), the peak is located at $n=\sim 4 \times 10^{3} \mathrm{~cm}^{-3}$ for all temperatures with a higher magnitude. For the $j=4 \rightarrow 3$ line, at low density, the amplitude increases with the temperature increase while the reverse is observed at high density because, under LTE conditions, the population of the $j=4$ level decreases with increasing temperature.

Comparing the bottom and top panels shows that the brightness temperature is at least 10 times higher for the pure rotational lines. For example at $T=10 \mathrm{~K}$ and $n=4 \times 10^{4} \mathrm{~cm}^{-3}$, the brightness temperature is $10.4 \mathrm{mK}$ for $j=8 \rightarrow 7$ and $0.8 \mathrm{mK}$ for $j_{F_{1}, F}=$ $8_{7,6} \rightarrow 76,5$.

\subsection{The CNCN abundance in cold dark clouds}

As mentioned in the introduction, CNCN was detected in L1544, L483 and tentatively in TMC-1 by Vastel et al. (2019); Agúndez et al. (2018b). These authors used the observational spectra along with LTE approximation to derive the $\mathrm{CNCN}$ abundance. Therefore, 

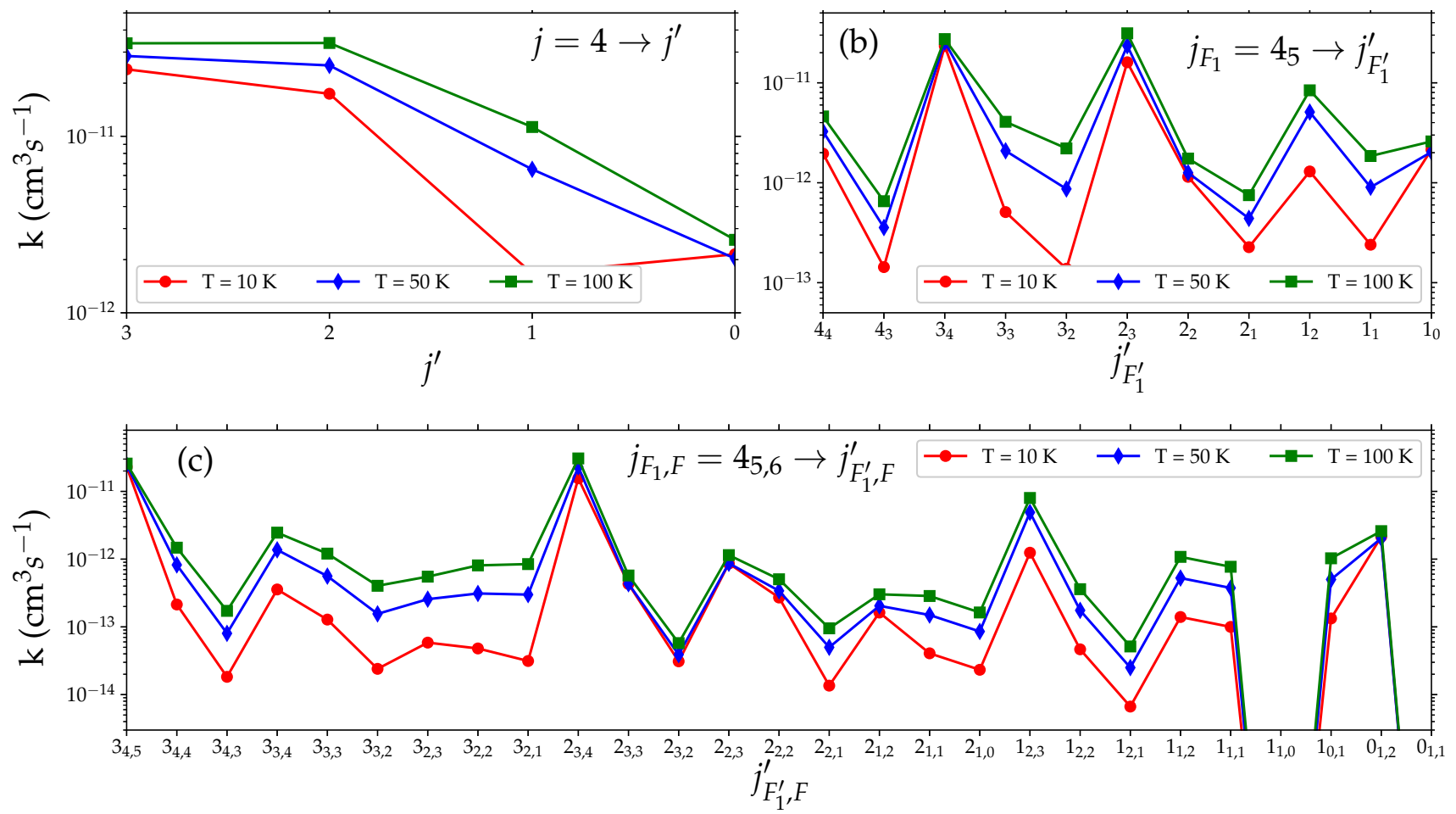

Figure 5. Downward rate coefficients of $\mathrm{CNCN}$ induced by collision with He. Panel (a) shows pure rotational transitions labeled as $10 \rightarrow j^{\prime}$ while panels (b) and (c) display transitions among the hyperfine structure due to the spin of one $\left(4_{5} \rightarrow j_{F_{1}^{\prime}}^{\prime}\right)$ and two $\left(4_{5,6} \rightarrow j_{F_{1}^{\prime}, F^{\prime}}^{\prime}\right)$ nitrogen nuclei, respectively. The data plotted in panel (b) are determined by summing the $k_{j, F_{1}, F \rightarrow j^{\prime}, F_{1}^{\prime}, F^{\prime}}$ over $F^{\prime}$.
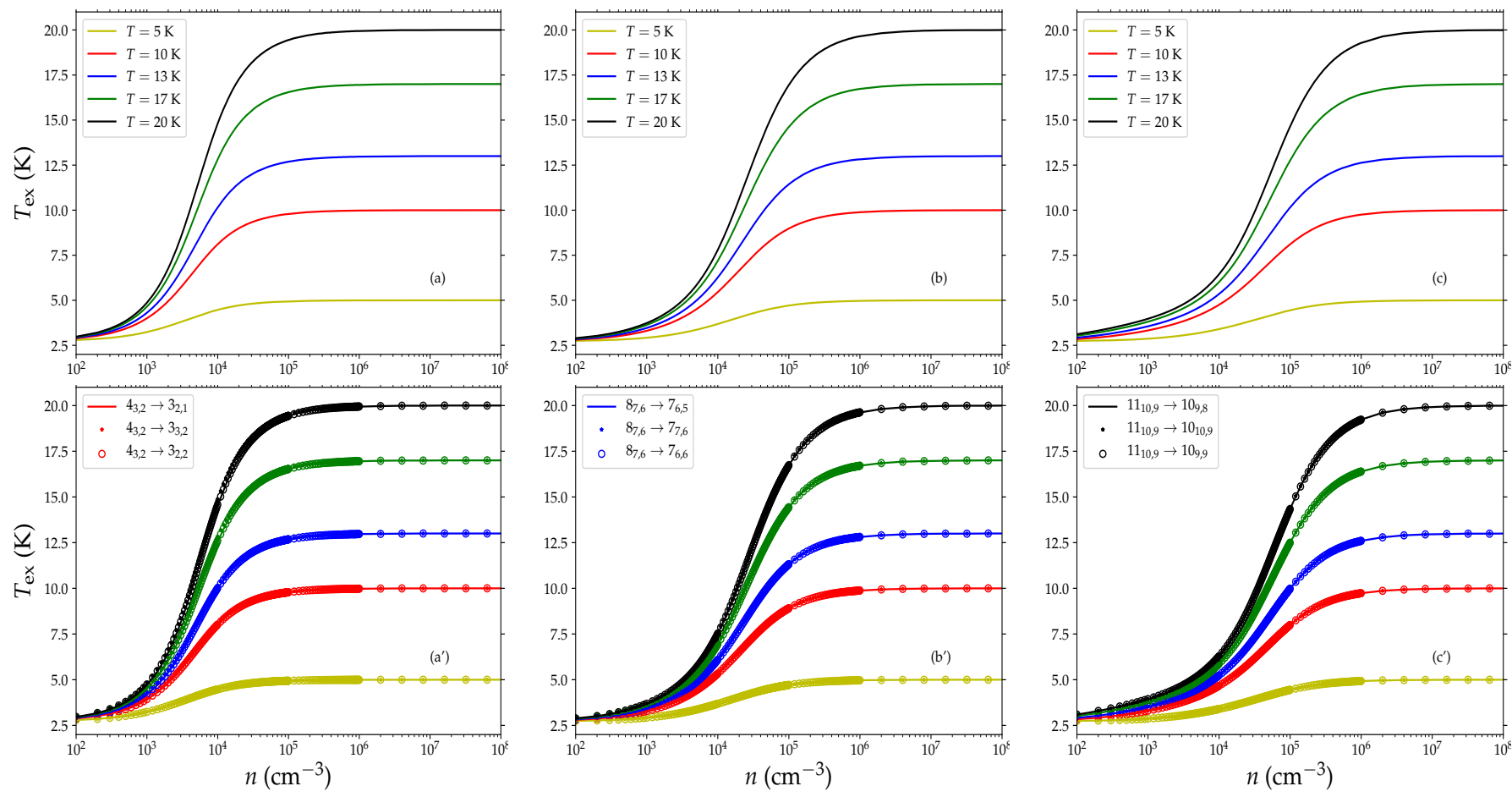

Figure 6. Variation of the $\mathrm{CNCN}$ excitation temperature for pure rotational (top panels) and hyperfine (bottom panels) emission lines as a function of the $\mathrm{H}_{2}$ volume density for selected temperatures (shown only in the top panels). Panels (a), (b) and (c) display the $j=4 \rightarrow 3,8 \rightarrow 7$ an $11 \rightarrow 10$ lines, respectively. Panels (a'), (b') and (c') display some hyperfine transitions as shown in the legend. The CNCN column density is fixed at $10^{12} \mathrm{~cm}^{-2}$. 

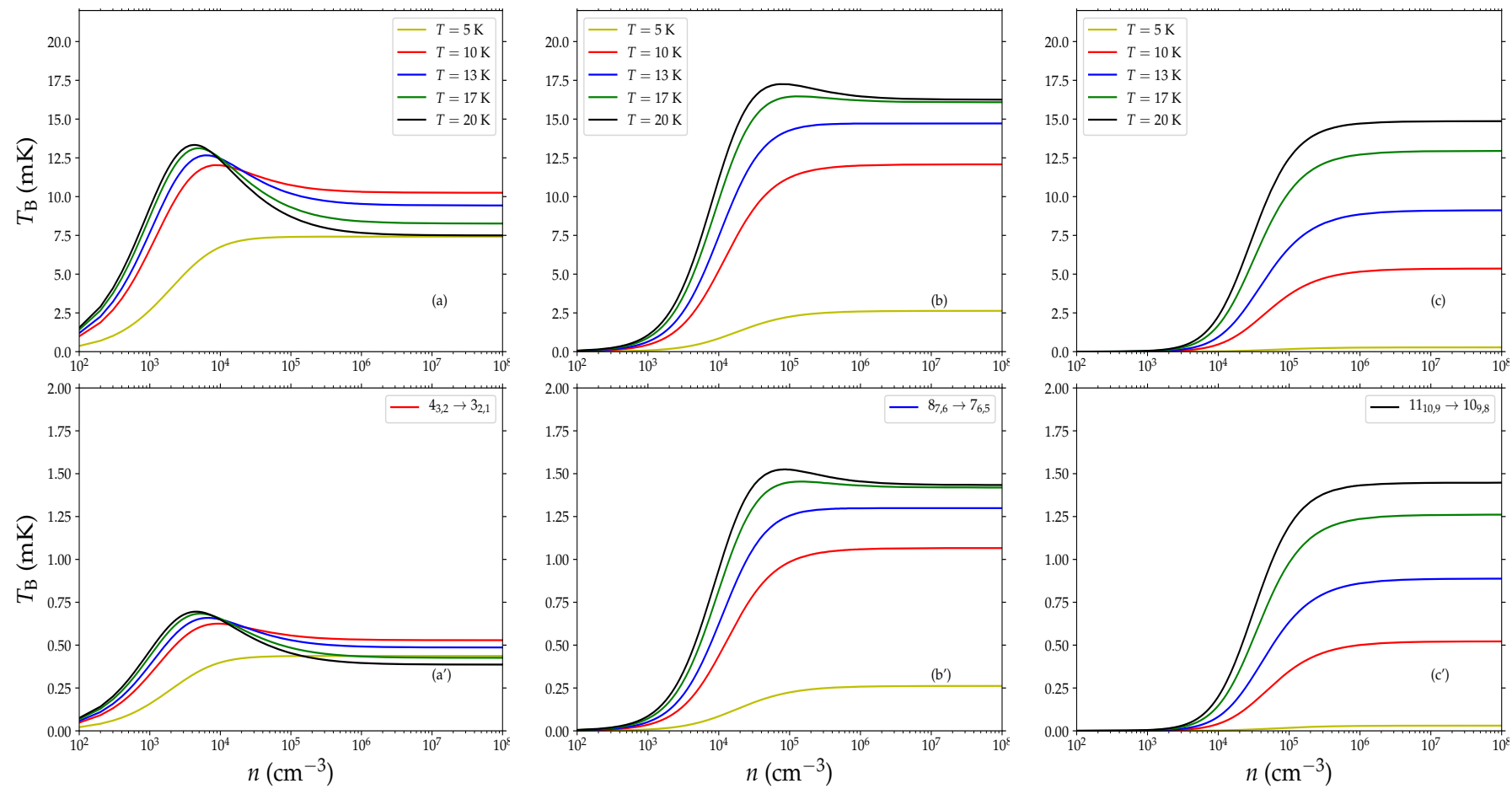

Figure 7. Variation of the $\mathrm{CNCN}$ brightness temperature for pure rotational (top panels) and hyperfine (bottom panels) emission lines as a function of the $\mathrm{H}_{2}$ volume density for selected temperatures. Panels (a), (b) and (c) show the $j=4 \rightarrow 3,8 \rightarrow 7$ and $11 \rightarrow 10$ lines, respectively. Panels (a'), (b') and (c') display the $j_{F_{1}, F}=4_{3,2} \rightarrow 3_{2,1}, 8_{7,6} \rightarrow 7_{6,5}$ and $11_{10,9} \rightarrow 10_{9,8}$ hyperfine transitions, respectively. The CNCN column density is fixed at $10^{12} \mathrm{~cm}^{-2}$.

it is of interest to assess the influence of our collision rate coefficients on the $\mathrm{CNCN}$ abundance estimation.

To model the CNCN spectra, we performed radiative transfer calculations for column densities ranging from $10^{11} \mathrm{~cm}^{-2}$ to $10^{13} \mathrm{~cm}^{-2}$ along with the parameters described in the beginning of this section. For all considered emission lines, we computed the integrated antenna temperatures $\left(W_{A}^{*}\right)$ as in Agúndez et al. (2018b) and the integrated intensites $(W)$ as in Vastel et al. (2019). The antenna parameters, $W_{A}^{*}=W \times B_{\text {eff }} / F_{\text {eff }}$, were derived using the the telescope beam efficiency $\left(B_{\text {eff }}\right)$ and forward efficiency $\left(F_{\text {eff }}\right)$ reported by Agúndez et al. (2018a).

As a first approach for deriving the $\mathrm{CNCN}$ abundance from the observational spectra from L1544 and L483 molecular clouds, we adopted the typical temperature and gas density of these cold dark clouds reported in the literature. For instance, Vastel et al. (2018) derived for $\mathrm{L} 1544, \mathrm{a} \mathrm{H}_{2}$ volume density of $(2 \pm 1) \times 10^{4} \mathrm{~cm}^{-3}$ and a gas kinetic temperature of $12 \mathrm{~K}$ while Agúndez et al. (2019) used a density of $\sim 3 \times 10^{4} \mathrm{~cm}^{-3}$ along with a temperature of $10 \mathrm{~K}$ for L 483 . Using these physical conditions, our model failed at reproducing the CNCN integrated intensities of Agúndez et al. (2018b); Vastel et al. (2019) within relative errors less than $\sim 30 \%$. This failure may be due to the simplicity of our radiative transfer model and/or the uncertainty on the physical conditions. In cold dark clouds, physical conditions may be heterogeneous. For example, Agúndez et al. (2019) estimated temperatures of $10 \mathrm{~K}$ and $15 \pm 2 \mathrm{~K}$ for the L483 protostar based on the $\mathrm{CO}$ and $\mathrm{CH}_{3} \mathrm{CCH}$ lines, respectively. For L1544, Quénard et al. (2017); Vastel et al. (2019) considered a density profile across the prestellar core in their model. This procedure allows to take into account the molecular depletion in the center of the core which is characterized by low temperature and high density.
Then, as a second approach, we released the constraints on the temperature and gas density. The best fit was determined by minimizing the $\chi^{2}$ parameter (eq. 11) $)^{2}$.

$$
\chi^{2}=\sum_{i}\left(\frac{W_{i}^{*, \mathrm{obs}}-W_{i}^{*, \mathrm{cal}}}{\sigma_{i}}\right)^{2}
$$

For the L483 molecular cloud, we derived a CNCN column density of $\sim 1.0 \times 10^{12} \mathrm{~cm}^{-2}$, a temperature in the $14 \mathrm{~K}-17 \mathrm{~K}$ range and a gas density of $(4.3-8.0) \times 10^{4} \mathrm{~cm}^{-2}$. The derived CNCN column density is lower than the value reported by Agúndez et al. (2018b) by a factor of $\sim 1.5$. The temperature we obtained is in agreement with that of Agúndez et al. (2019) estimated using $\mathrm{CH}_{3} \mathrm{CCH}$, suggesting that $\mathrm{CNCN}$ is present in the inner region of L483. Concerning the gas density, our result is a little higher than the expected value of $\sim 3 \times 10^{4} \mathrm{~cm}^{-3}$ but still in reasonable agreement.

For the L1544 prestellar core, our model fails at reproducing the observations of Vastel et al. (2019) despite the release of all constraints on the physical parameters. Indeed, no set of inputs can reproduce simultaneously the $j=8 \rightarrow 7$ and $10 \rightarrow 9$ lines detected in L1544 when using 20-30\% as convergence threshold with respect to measurements. However, Vastel et al. (2019) constrained the $\mathrm{CNCN}$ excitation temperature of the $j=8 \rightarrow 7$ and $10 \rightarrow 9$ lines between $13 \mathrm{~K}$ and $17 \mathrm{~K}$ and derived a column density of $(6.0-6.6) \times 10^{11} \mathrm{~cm}^{-2}$. Such a $T_{\mathrm{ex}}$ range is consistent with excitation calculations with $n \geq 10^{5} \mathrm{~cm}^{-3}$ or $T \geq 20 \mathrm{~K}$. These values are much higher than what was derived previously and probably reflect

$2 W^{*}$,obs and $W^{* \text {,cal }}$ are the integrated antenna temperatures and integrated intensities derived from the observations and RADEX calculations, respectively. The index $i$ stands for the number of observed lines and $\sigma$ refers to the uncertainty on the measurements. 
some limitations in our radiative transfer models. However, the rate coefficients presented in this work can be implemented in a more robust radiative transfer code, that takes into account the complex nature of L1544, to improve the results.

To estimate the CNCN abundance in TMC-1 using our model, we stacked the integrated antenna temperature corresponding to four emission lines (from $j=8 \rightarrow 7$ to $11 \rightarrow 10$ ) as done by Agúndez et al. (2018b). In fact, these authors provided the peak emission resulted from the stacking since the detection was qualified as tentative due to low signal-to-noise ratios. Note that the physical conditions cannot be probed using the single line resulting from the stacking. Therefore, the gas density and temperature were set to $4.4 \times 10^{4} \mathrm{~cm}^{-3}$ and $10 \mathrm{~K}$, respectively, as usually done in the literature for TMC-1. We derived a column density of $6 \times 10^{11} \mathrm{~cm}^{-2}$ which best reproduces the observations of Agúndez et al. (2018b). The CNCN abundance obtained using our set of data is 1.5 times lower than the result previously reported by the authors mentioned above.

\section{CONCLUSION}

A new PES, calculated at the $\operatorname{CCSD}(\mathrm{T})-\mathrm{F} 12 /$ aug-cc-pVTZ level of theory, was used to investigate the collisional excitation of isocyanogen due to helium impact. State-to-state rotational inelastic cross-sections were derived using the exact close-coupling quantum mechanical method for total energies up to $800 \mathrm{~cm}^{-1}$. By thermally averaging these data in the Maxwell-Boltzmann velocity distribution, we obtained downward rate coefficients involving the 30 low-lying rotational levels for temperatures up to $150 \mathrm{~K}$. Thereafter, we fully resolved the $\mathrm{CNCN}$ hyperfine structure using the IOS based approach.

Subsequently, we carried out non-LTE radiative transfer calculations using the RADEX code. Using our set of data, we were able to reproduce fairly well the integrated antenna temperatures measured by Agúndez et al. (2018b) for L483 and TMC-1 with CNCN column densities of $\sim 1.1 \times 10^{12} \mathrm{~cm}^{-2}$ and $6 \times 10^{11} \mathrm{~cm}^{-2}$, respectively. The newly derived column densities in the two sources are $\sim 1.5$ times smaller than those reported by Agúndez et al. (2018b). Calling back the CNCN column density derived by Vastel et al. (2019) for L1544, we remark that this molecule has quite similar column densities $\left(6 \times 10^{11} \mathrm{~cm}^{-2}-6.6 \times 10^{11} \mathrm{~cm}^{-2}\right)$ in TMC-1 and L1544 whereas in L483, the CNCN column density is almost doubled $\left(\sim 1.1 \times 10^{12} \mathrm{~cm}^{-2}\right)$.

As discussed in the introduction, the use of the $\mathrm{CNCN}$ abundance is a manner to roughly estimate that of NCCN. Therefore, we report NCCN column densities of $\sim 8 \times 10^{13} \mathrm{~cm}^{-2}$ and $\sim 4 \times 10^{13} \mathrm{~cm}^{-2}$ for L483 and TMC-1, respectively. Further nonLTE modeling of the $\mathrm{HNCCN}^{+}, \mathrm{HCCCN}$ and $\mathrm{HCCNC}$ abundances are required to draw a more robust conclusion for the abundance of $\mathrm{NCCN}, \mathrm{CNCN}$ and $\mathrm{HNCCN}^{+}$. Since accurate collision rate coefficients for $\mathrm{HCCCN}$ and $\mathrm{HCCNC}$ are available in the literature (Bop et al. 2021) we can expect to have soon a better insight into the abundance of NCCN in space. Such improvements will encourage to revise the chemistry of $\mathrm{NCCN}, \mathrm{CNCN}$ and $\mathrm{HNCCN}^{+}$.

\section{ACKNOWLEDGEMENTS}

The authors acknowledge the Programme National "Physique et Chimie du Milieu Interstellaire" (PCMI) of Centre National de la Recherche Scientifique (CNRS)/Institut National des Sciences de
l'Univers (INSU) with Institut de Chimie (INC)/Institut de Physique (INP) co-funded by Commissariat a l'Energie Atomique (CEA) and Centre National d'Etudes Spatiales (CNES). F.L. acknowledges the Institut Universitaire de France.

\section{DATA AVAILABILITY}

The data underlying this article are available in the article and in its online supplementary material.

\section{REFERENCES}

Agúndez M., Cernicharo J., Guélin M., 2014, Astronomy \& Astrophysics, 570, A45

Agúndez M., et al., 2015, Astronomy \& Astrophysics, 579, L10

Agúndez M., Marcelino N., Cernicharo J., Tafalla M., 2018a, Astronomy \& Astrophysics, 611, L1

Agúndez M., Marcelino N., Cernicharo J., 2018b, The Astrophysical Journal Letters, 861, L22

Agúndez M., Marcelino N., Cernicharo J., Roueff E., Tafalla M., 2019, Astronomy \& Astrophysics, 625, A147

Alexander M. H., Manolopoulos D. E., 1987, The Journal of chemical physics, 86, 2044

Arthurs A., Dalgarno A., 1960, in Proceedings of the Royal Society of London A: Mathematical, Physical and Engineering Sciences. pp 540551

Balança C., Scribano Y., Loreau J., Lique F., Feautrier N., 2020, Monthly Notices of the Royal Astronomical Society, 495, 2524

Ben Abdallah D., Najar F., Jaidane N., Dumouchel F., Lique F., 2012, Monthly Notices of the Royal Astronomical Society, 419, 2441

Ben Abdallah D., Mogren Al Mogren M., Dhaif Allah Al Harbi S., Hochlaf M., 2018, The Journal of Chemical Physics, 149, 064305

Bop C. T., 2019, Monthly Notices of the Royal Astronomical Society, 487, 5685

Bop C. T., Boye Faye N., Hammami K., Jaïdane N., 2017a, The Journal of Physical Chemistry A, 121, 7854

Bop C. T., Hammami K., Niane A., Faye N., Jaïdane N., 2017b, Monthly Noticies of the Royal Astronomical Society, 465, 1137

Bop C. T., Faye N., Hammami K., 2018, Monthly Notices of the Royal Astronomical Society, 478, 4410

Bop C. T., Lique F., Faure A., Quintas-Sánchez E., Dawes R., 2021, Monthly Notices of the Royal Astronomical Society, 501, 1911

Botschwina P., 1994, Chemical Physics Letters, 225, 480

Boys S. F., Bernardi F. d., 1970, Molecular Physics, 19, 553

Cernicharo J., Marcelino N., Agúndez M., Bermúdez C., Cabezas C., Tercero B., Pardo J., 2020, arXiv preprint arXiv:2009.07686

Dunning Jr T. H., 1989, The Journal of chemical physics, 90, 1007

Endres C. P., Schlemmer S., Schilke P., Stutzki J., Müller H. S., 2016, Journal of Molecular Spectroscopy, 327, 95

Goldflam R., Green S., Kouri D., 1977, The Journal of Chemical Physics, 67,4149

Hutson J., Green S., 1994, Collaborative computational project

Knizia G., Adler T. B., Werner H.-J., 2009, The Journal of Chemical Physics, 130, 054104

Lique F., Toboła R., Kłos J., Feautrier N., Spielfiedel A., Vincent L., Chałasiński G., Alexander M., 2008, Astronomy \& Astrophysics, 478, 567

Lique F., Kłos J., Hochlaf M., 2010, Physical Chemistry Chemical Physics, 12,15672

Neufeld D. A., Green S., 1994, The Astrophysical Journal, 432, 158

Petrie S., Millar T., Markwick A., 2003, Monthly Notices of the Royal Astronomical Society, 341, 609

Puzzarini C., Cazzoli G., 2009, Journal of Molecular Spectroscopy, 256, 53

Quénard D., Vastel C., Ceccarelli C., Hily-Blant P., Lefloch B., Bachiller R., 2017, Monthly Notices of the Royal Astronomical Society, 470, 3194 
Roueff E., Lique F., 2013, Chemical reviews, 113, 8906

Stroh F., Winnewisser M., 1989, Chemical Physics Letters, 155, 21

Van der Tak F., Black J. H., Schöier F., Jansen D., van Dishoeck E. F., 2007, Astronomy \& Astrophysics, 468, 627

Vastel C., et al., 2018, Monthly Notices of the Royal Astronomical Society, 478,5514

Vastel C., Loison J., Wakelam V., Lefloch B., 2019, Astronomy \& Astrophysics, 625, A91

Werner H.-J., et al., 2010, See http://www. molpro. net

Wernli M., Wiesenfeld L., Faure A., Valiron P., 2007, Astronomy \& Astrophysics, 464, 1147 